\title{
PROPOLIS IMPROVED THE HEPATIC ARCHITECTURE BY CONTROLLING STAT-3 AND STAT-5 PHOSPHORYLATION, AND SURVIVIN EXPRESSION IN A MOUSE MODEL OF LIVER FIBROSIS
}

\author{
Eman Abdo Sayed; Hanan Waly; Khadiga Abdel-Hameed Hassan; \\ Gamal Badr*
}

Zoology Department, Faculty of Science, Assiut University, Assiut, Egypt

\begin{abstract}
Article History:
Received: 28 March 2020

Revised: 11 May 2020

Accepted: 12 May 2020

Published Online:

16 May 2020

Keywords:

Apoptosis

Inflammation

Liver fibrosis

Oxidative stress

Propolis

*Correspondence:

Gamal Badr

Zoology Department

Faculty of Science

Assiut University

Assiut, Egypt

E-mail:

gamal.badr@aun.edu.eg

ABSTRACT

Propolis has several biological/pharmacological properties. The current study investigated the potential hepatoprotective benefits of propolis in $\mathrm{CCl}_{4}$-treated mice. Three groups of male $\mathrm{BALB} / \mathrm{c}$ mice ( $n=15 /$ group) were used in the current study: group I comprised the control mice, groups II was intraperitoneally injected with $\mathrm{CCl}_{4}\left(1.0 \mathrm{~mL}\right.$ of $10 \% \mathrm{CCl}_{4}$ dissolved in olive oil $/ \mathrm{kg}$ body weight, twice/week for six weeks) for inducing liver fibrosis, group III was treated with $\mathrm{CCl}_{4}$ as in group II and then supplemented orally with the ethanol-soluble derivative of propolis $(100 \mathrm{mg} / \mathrm{kg}$ body weight/day) for additional four weeks. The antifibrotic effects of propolis were assessed by histological analysis, Western blotting, flow cytometry, and ELISA. The results indicated that the $\mathrm{CCl}_{4}$-treated mice exhibited histopathological alterations in the liver architecture with an increase in the numbers of Kupffer cells, a significant increase in the lymphocytes apoptosis and in the plasma nitric oxide, reactive oxygen species, C-reactive protein, and platelet derived growth factor levels, and a significant decrease in the plasma total glutathione level, as compared with the control group. The liver of $\mathrm{CCl}_{4}$-treated mice also exhibited a significant increase in the expression of collagen and survivin, upregulation of signal transducer and activator of transcription 3 (STAT3) phosphorylation, and downregulation of STAT5 phosphorylation. Interestingly, propolis abrogated significantly the hepatic collagen deposition, inflammatory signals, and oxidative stress, and improved the hepatic architecture in $\mathrm{CCl}_{4}$-treated mice nearly to the normal architecture observed in the control mice. In conclusion, our findings demonstrate the potential hepatoprotective effects of propolis in alleviating the liver fibrosis.
\end{abstract}

\section{INTRODUCTION}

Hepatic diseases still represent a problem for the economics of healthcare worldwide. Liver fibrosis is a wound-healing process that occurs in response to chronic hepatic injury of a variety of aetiologies and eventually progresses to liver cirrhosis following persistent inflammation and 
fibrogenesis ${ }^{[1]}$. The most common causes of hepatic fibrosis are untreated viral hepatitis B and C infection, alcoholic liver diseases, non-alcoholic fatty liver diseases, parasites, autoimmunity, and less commonly some drugs ${ }^{[2]}$. Fibrosis is the excessive accumulation of extracellular matrix (ECM) proteins in the liver, as a result of chronic hepatic injury ${ }^{[2]}$. Hepatic stellate cells (HSCs), the main hepatic cells implicated in the accumulation of ECM proteins, are activated by several cytokines, such as interleukin (IL)-1 $\beta$, IL-6, and transforming growth factor beta, as well as reactive oxygen species $(\mathrm{ROS})^{[3]}$. Until now, no treatment has successfully stopped the development of hepatic diseases. In addition, the newly developed medications that are used to treat chronic hepatic diseases have many side effects ${ }^{[4]}$. Therefore, it was essential to search for suitable natural drugs from herbal and animal sources that could replace the chemical ones ${ }^{[4,5]}$.

Carbon tetrachloride $\left(\mathrm{CCl}_{4}\right)$ is one of xenobiotics that have been reported to cause acute and chronic tissue injuries, and is a well-established hepatotoxin, so it has been used extensively to study the hepatotoxicity in animal models by initiating lipid peroxidation, in addition to liver pathogenesis ${ }^{[5,6]}$. The $\mathrm{CCl}_{4}$ has been shown to cause acute hepatotoxicity with necrotic and apoptotic hepatocellular injury, and loss of liver function. Various lines of evidence indicate that damage to the liver can be avoided by inhibiting the oxidative stress and the inflammatory response ${ }^{[6]}$.

Nature has presented to humanity the gift of biological and cultural diversity of natural product for healing practices. One of the natural products commonly used in traditional medicine without proper consideration of its safety is bee propolis. Propolis is one of the most promising natural products presenting not only therapeutic action, but also a prophylactic one. It has therapeutic properties such as antimicrobial, anti-inflammatory, immunomodulatory, antioxidant, and antitumour activities $^{[7]}$. In addition, ethanolic extract of propolis showed anti-inflammatory properties in both chronic and acute inflammation ${ }^{[7]}$, which may exert protective effects against hepatotoxicity. Bee propolis contains large amounts of antioxidant compounds, such as caffeic acid, ferulic acid, and caffeic acid phenethyl ester. Chemically, propolis obtained from different areas of the world is constituted by $50-60 \%$ of resin, $30-40 \%$ of wax, $5-10 \%$ of essential oils, and $5 \%$ of pollen, in addition to microelements ${ }^{[7]}$. Therefore, the current study investigated the potential hepatoprotective effects of propolis against $\mathrm{CCl}_{4}$-induced liver fibrosis in mice.

\section{MATERIAL AND METHODS Preparation of propolis}

Honey Spring propolis was purchased from Etman Hives, Shabshir Al Hissah, Gharbia, Egypt. Propolis was characterized at our laboratory as previously described by Jerz et $a l .{ }^{[8]}$. Abundant data from our laboratory using various animal models indicated that a daily dose of $50-250 \mathrm{mg} / \mathrm{kg}$ body weight of ethanol-soluble derivative of bee propolis does not cause toxic effects. Therefore, this dose is considered safe, and hence an optimal concentration $(100 \mathrm{mg} / \mathrm{kg}$ body weight) of ethanol-soluble derivative of bee propolis was used in the current study for the treatment of mice with liver fibrosis.

\section{Carbon tetrachloride $\left(\mathrm{CCl}_{4}\right)$-induced liver fibrosis}

The $\mathrm{CCl}_{4}$ was obtained from Sigma Chemicals Co. (St. Louis, MO, USA). For the induction of chronic liver fibrosis, animals received intraperitoneal (i.p.) injections of $\mathrm{CCl}_{4}\left(1.0 \mathrm{~mL}\right.$ of $10 \% \mathrm{CCl}_{4}$ dissolved in olive oil $/ \mathrm{kg}$ body weight) twice/week for 6 weeks.

\section{Experimental design and doses}

In this study, 45 adult male mice BALB/c mice (Mus musculus) weighing (25-30 g) were used. The mice were bought from Institute of Theodor Bilharz, Giza, Egypt. Mice were housed in cages and kept in 
a room temperature at $25 \pm 5^{\circ} \mathrm{C}$ with normal 12 hours light/12 hours dark cycle. They had ad libitum access to pelleted diet and water for one week for acclimatisation. The research procedures used in the current study was approved by the Animal Care and Use Committee of the Faculty of Science, Assiut University. After one week of acclimatisation, animals were randomly allotted into three experimental groups ( $\mathrm{n}=15 /$ group): control group (group I), $\mathrm{CCl}_{4}{ }^{-}$ treated group (group II), and $\mathrm{CCl}_{4}$-treated group that was treated with propolis (group III, $\mathrm{CCl}_{4}+$ propolis-treated group). Liver fibrosis was induced in groups II and III as described in the previous section. After induction of liver fibrosis, group III was then supplemented orally with $100 \mu \mathrm{L}$ of $50 \%$ ethanol-soluble derivative of bee propolis $(100 \mathrm{mg} / \mathrm{kg}$ body weight/day) for additional four weeks. The control group was injected with vehicles alone $(1.0 \mathrm{~mL}$ of olive oil $/ \mathrm{kg}$ body weight, twice/week for six weeks). In addition, groups I and II received $100 \mu \mathrm{L}$ of $50 \%$ ethanol per day (as a vehicle) by oral gavage for additional four weeks.

\section{Blood collection and analysis}

Whole blood was collected from the abdominal aorta in heparinised tubes. The blood was centrifuged at $3000 \times g$ for 20 minutes using a bench top centrifuge (Anke TGL-16B; Hinotek Lab, Ningbo, China) to remove red blood cells and recover plasma. The Plasma samples were collected by using dry Pasteur pipettes, and stored at $-20^{\circ} \mathrm{C}$ before further use. Peripheral blood mononuclear cells (PBMCs) were separated by using the Ficoll gradient method for flow cytometry analysis.

\section{Histopathological and Immunohisto- chemistry analyses}

Mouse liver tissues were fixed with $10 \%$ neutral formalin, dehydrated, and further embedded with paraffin. Paraffin-embedded liver samples were sectioned into $5 \mu \mathrm{m}$-thin slices and stained with haematoxylin and eosin (H\&E), Masson's trichrome, or Sirius red. Sections were examined under light microscopy by an experienced pathologist. To detect Kupffer cells distribution in liver samples, anti-CD68 antibodies (sc-20060, SANTA CRUZ, Paris, France), were used as primary antibodies as previously described by Hidaka et al. ${ }^{[9]}$.

\section{Measuring of oxidative stress markers and C-reactive protein (CRP)}

Plasma ROS levels were measured using 2,7-dichlorodihydrofluorescein diacetate at laboratory of haematology and medical analysis in Monufia University Hospital (Monufia, Egypt), as previously described by Eruslanov and Kusmartsev ${ }^{[10]}$. The plasma concentration of nitrite $\left(\mathrm{NO}_{2}{ }^{-}\right)$was determined by the Griess reaction, as an indicator of nitric oxide (NOx) production. Briefly, $100 \mu \mathrm{L}$ of samples and $100 \mu \mathrm{L}$ of Griess reagent a mixture of $2 \%$ sulphanilamide in $5 \%$ phosphoric acid and $0.2 \% \mathrm{~N}$-(1-naphthyl) ethylenediamine hydrochloride] were mixed in 96well ELISA (enzyme-linked immunosorbent assay) plates. Absorbance was measured at $550 \mathrm{~nm}$, and the levels of $\mathrm{NO}_{2}{ }^{-}$ were determined using a standard curve of $\mathrm{NaNO}_{2}$. The level of plasma total glutathione was determined by the Cayman Chemical assay kit (Ann Arbor, MI, USA) according to the manufacturer's instruction and as previously described by Ono et $a l^{[11]}$. Plasma CRP was estimated as previously described by Sin et al. ${ }^{[12]}$.

\section{Measuring of platelet-derived growth factor (PDGF) level}

The PDGF level was measured in plasma using rabbit anti-mouse anti-PDGF antibody (ab178409) in ELISA sets (Indirect ELISA) according to the protocol that is provided by the supplier (Abcam, Cambridge, United Kingdom).

\section{Western blots Analysis}

Whole-tissue lysates were prepared from the liver tissues isolated from control and treated mice. Liver tissues were incubated in radioimmunoprecipitation assay buffer for 30 minutes. Following centrifugation at 
$16000 \times g$ for 15 minutes at $4^{\circ} \mathrm{C}$, the protein concentration of each supernatant was determined using a protein assay kit (Bio-Rad, Hercules, CA, USA), and the lysates were then stored at $-20^{\circ} \mathrm{C}$ for Western blot analysis. The primary rabbit anti-mouse antibodies were purchased from Abcam for collagen I (ab34710), survivin (ab469), phosphoSTAT3 (phospho Y705, ab76315), phosphoSTAT5 (phospho Y694, ab32364), total STAT3 (ab68153), total STAT5 (ab16276), $\beta$-actin (ab8227), and used in the current study for Western blot analysis as previously described ${ }^{[13] .}$

\section{Flow cytometry analysis}

The flow cytometry was used to determine the percentage of apoptotic lymphocytes. The mouse PBMCs were separated using the Ficoll gradient method. Cells were washed with phosphate-buffered saline (PBS), counted using the trypan blue exclusion test, and cultured in R-10 medium (complete RPMI 1640 medium supplemented with $10 \%$ foetal calf serum, $2.0 \mathrm{mmol}$ L-glutamine, $100 \mathrm{IU} / \mathrm{mL}$ penicillin, $\quad 100 \mu \mathrm{g} / \mathrm{mL}$ streptomycin, $1.0 \mathrm{mmol}$ sodium pyruvate, and $50 \mu \mathrm{mol}$ 2-mercaptoethanol) for one hour before being subjected to flow cytometry analysis to monitor the percentage of lymphocytes undergoing apoptosis. In order to distinguish between viable, early apoptotic, and late apoptotic cells, PBMCs were washed and incubated in PBS containing 30\% human $\mathrm{AB}$ serum for 30 minutes at $4^{\circ} \mathrm{C}$ prior to staining with Annexin V-fluorescein isothiocyanate (FITC) and propidium iodide (PI) for 15 minutes and at $25^{\circ} \mathrm{C}$ using a commercial kit (Abcam), according to the manufacturer's instructions. Cells were then analysed by flow cytometry using a FACS Calibur flow cytometer (BDPharmingen, San Jose, CA, USA) within one hour of staining to determine the percentage of cells undergoing apoptosis. After the gates were set to include only lymphocytes according to the forward scatter/side scatter (FSC/SSC), $10^{4}$ events per sample were collected and analysed.

\section{Statistical analysis}

The data were tested for normality using Anderson-Darling tests and variance homogeneity prior to further statistical analysis. The data were normally distributed and are expressed as the means \pm their standard errors (SEM). Significant differences between groups were analysed using one-way analysis of variance (for more than two groups) followed by Tukey's post-hoc test using GraphPad Prism software version 5 (GraphPad software, Inc., San Diego, CA, USA). Differences with $P$ values $<0.05$ were considered statistically significant.

\section{RESULTS}

\section{Propolis alleviated the $\mathrm{CCl}_{4}$-induced histological alterations and fibrosis in the liver}

According to the H\&E staining, the liver sections of the control group displayed normal histological features of hepatic cells and normal central veins (Figure 1a). The liver sections of the $\mathrm{CCl}_{4}$ treated group showed massive, diffuse, and progressive histological alterations, in addition to loss of architecture with vacuolar degeneration and necrosis of hepatocytes, fatty changes in hepatocytes, and dilated congested central veins (Figure 1b). The $\mathrm{CCl}_{4}+$ propolis-treated group showed disappearance of most of the histological alterations to be more or less similar to that of the control group (Figure 1c). To monitor collagen deposition in the liver sections of the $\mathrm{CCl}_{4}$-treated group, both Masson trichrome and Sirius Red staining methods were used for confirmation. Compared with the control group, the $\mathrm{CCl}_{4}$-treated group exhibited abundant collagen fibres surrounding the central vein (Figures $1 \mathrm{e}$ and $\mathrm{h}$ ). In the $\mathrm{CCl}_{4}$ +propolis-treated group, the liver sections exhibited a decrease in the collagen fibres surrounding the central vein as compared with the $\mathrm{CCl}_{4}$-treated group (Figures 1f and i). 
Control
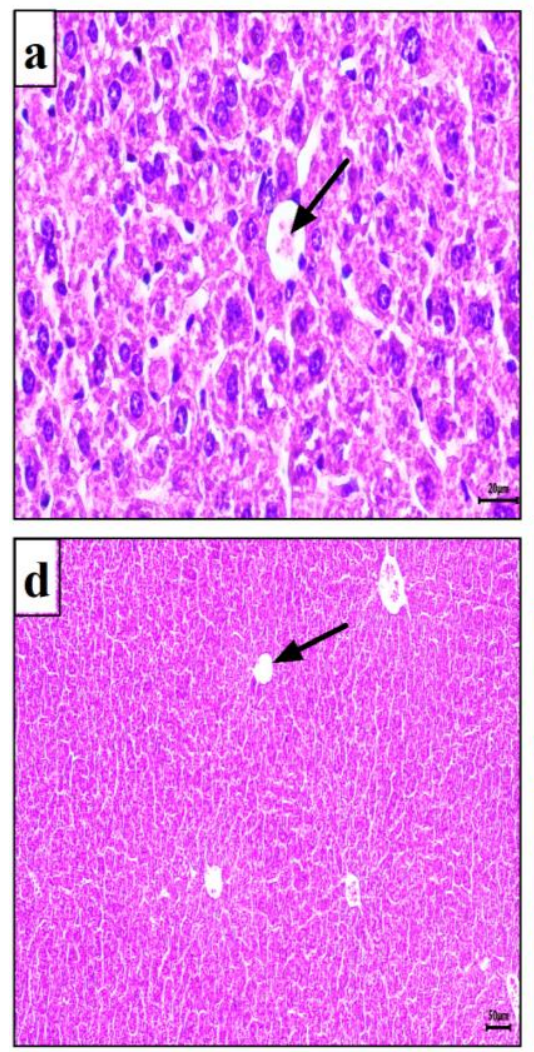

g

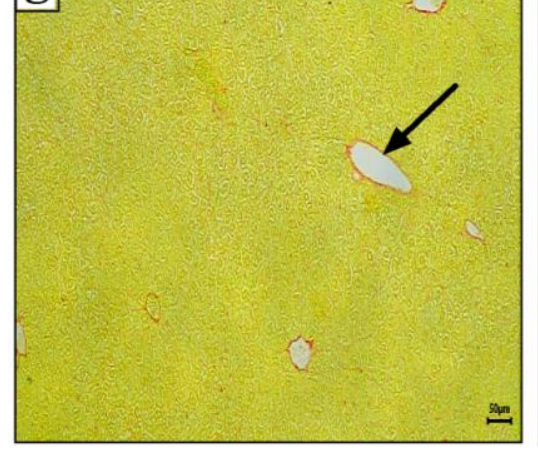

$\mathrm{CCl}_{4}$
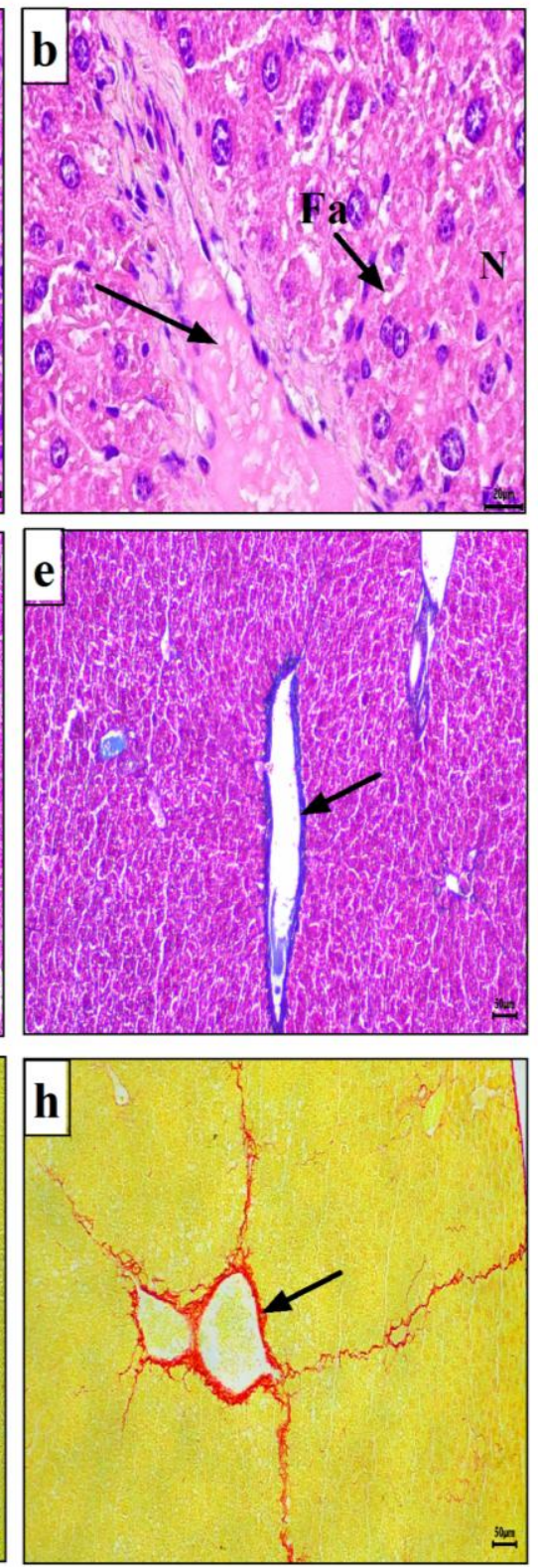

$\mathrm{CCl}_{4}+$ Propolis
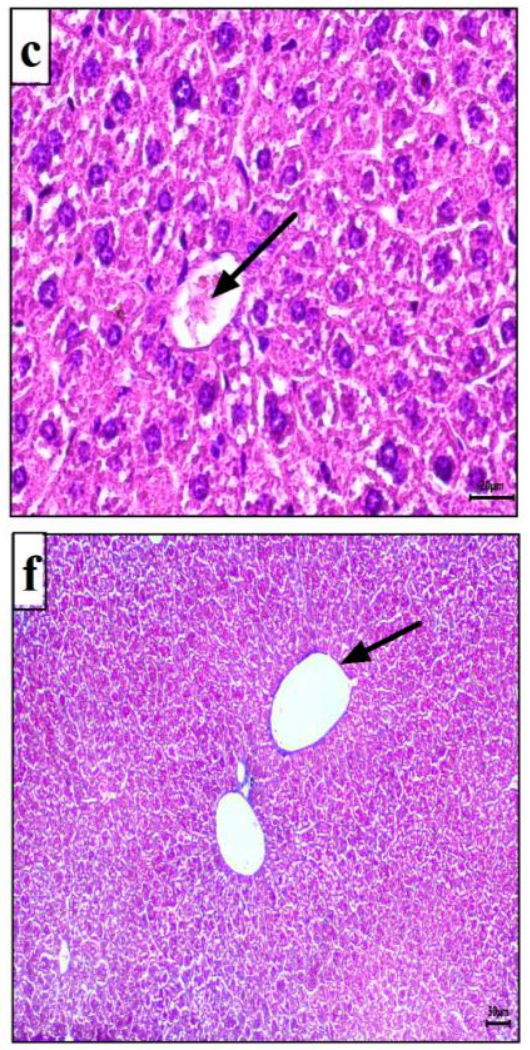

i

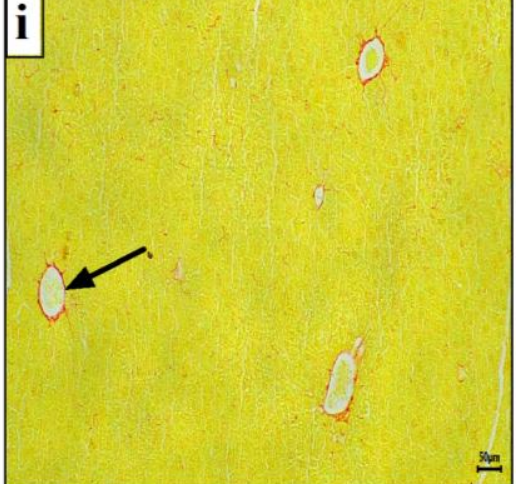

Figure 1: Photomicrographs of liver sections from control, $\mathrm{CCl}_{4}$-treated, and $\mathrm{CCl}_{4}+$ propolistreated groups stained with haematoxylin and eosin (H\&E) (a-c), Masson's trichrome (d-f), or Sirius red for collagen deposition (g-i). The images (a-c) are at $\times 400$ magnification (scale bar $=20 \mu \mathrm{m})$, but the images $(\mathbf{d}-\mathbf{i})$ are at $\times 100$ magnification (scale bar $=50 \mu \mathrm{m})$, and are all representative of samples from mice in each group. $\mathrm{Fa}$ : fat droplets, $\mathrm{N}$ : necrosis, arrows: central vein.

Propolis supplementation restored the number of Kupffer cells in $\mathrm{CCl}_{4}$-induced liver fibrosis

The Kupffer cell was monitored through both reacting with anti-CD86 antibodies and the irregular cell membrane enclosed nearly a triangular nucleus. The liver sections of the control group (Figures 2a and d) and the $\mathrm{CCl}_{4}$-treated group (Figures $2 \mathrm{~b}$ and e) revealed that small and high Kupffer cells numbers, respectively. Interestingly, the number of Kupffer cells in the $\mathrm{CCl}_{4}$ treated group was decreased by propolis treatment (Figures 2c and f). 

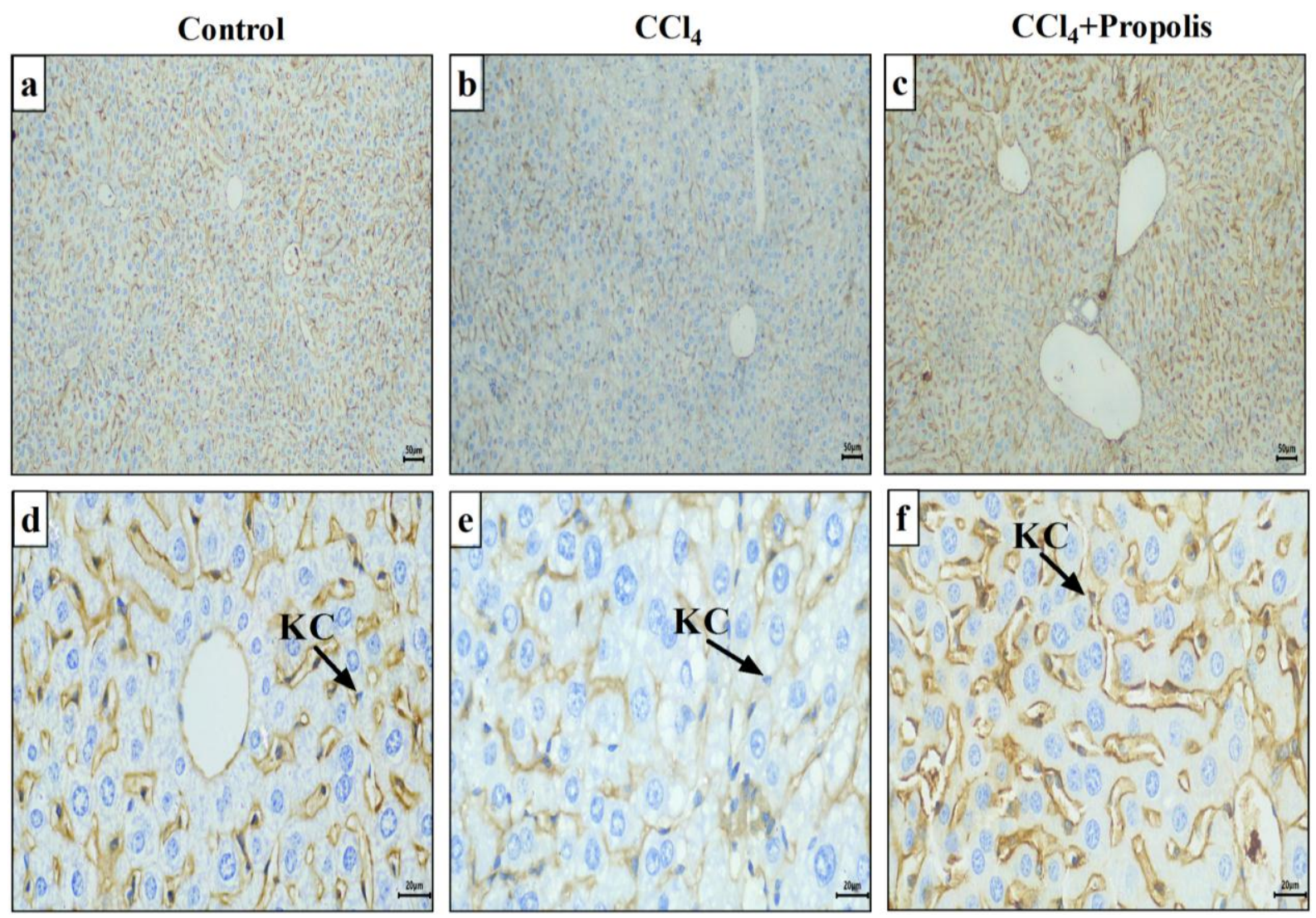

Figure 2: Photomicrographs of immunohistochemical analysis using anti-CD68 antibodies to determine the Kupffer cells distribution in the liver sections of the control (a and d), $\mathrm{CCl}_{4}$ treated (b and $\mathbf{e}$ ), and $\mathrm{CCl}_{4}+$ propolis-treated (c and $\mathbf{f}$ ) groups. a-c: magnification $=\times 100$ and scale bar $=50 \mu \mathrm{m}, \mathbf{d - f}$ : magnification $=\times 400$ and scale bar $=50 \mu \mathrm{m}, \mathrm{KC}$ : Kupffer cells.

Treatment with propolis decreased the lymphocytes apoptosis in $\mathrm{CCl}_{4}$-induced liver fibrosis

The PBMCs isolated from control, $\mathrm{CCl}_{4}$ treated, and $\mathrm{CCl}_{4}+$ propolis-treated mice were stained with PI/Annexin $\mathrm{V}$ and analysed by flow cytometry to determine the percentages of viable cells (lower left quadrant), early apoptotic cells (lower right quadrant), and late apoptotic cells (upper right quadrant) (Figure 3a). The data from one representative experiment are presented in the dot plot. The percentage of apoptotic lymphocytes (early and late apoptotic cells) increased markedly to $68 \%$ in the $\mathrm{CCl}_{4}$ treated group $(12 \%$ only in the control group), while it decreased markedly to $31 \%$ in $\mathrm{CCl}_{4}+$ propolis-treated mice (Figure $3 \mathrm{a}$ ). The pooled data of five mice from each group indicated that treatment of mice with fibrotic liver with propolis rescued significantly $(P<0.05)$ lymphocytes from apoptosis (Figure $3 b$ ).

\section{Supplementation with propolis modulated the oxidative stress and inflammatory markers in plasma of $\mathbf{C C l}_{4}$-treated mice}

The levels of CRP (Figure 4a), NOx (Figure 4b), ROS (Figure 4d), and PDGF (Figure 4e) in the plasma were significantly increased $(P<0.05)$ in the $\mathrm{CCl}_{4}$-treated mice compared with the control mice. Interestingly, supplementation of $\mathrm{CCl}_{4}$ treated mice with propolis restored significantly $(P<0.05)$ the plasma levels of CRP, NOx, ROS and PDGF compared with those in $\mathrm{CCl}_{4}$-treated mice. In addition, the level of total glutathione was significantly decreased $(P<0.05)$ in the plasma of $\mathrm{CCl}_{4}$-treated mice compared with 

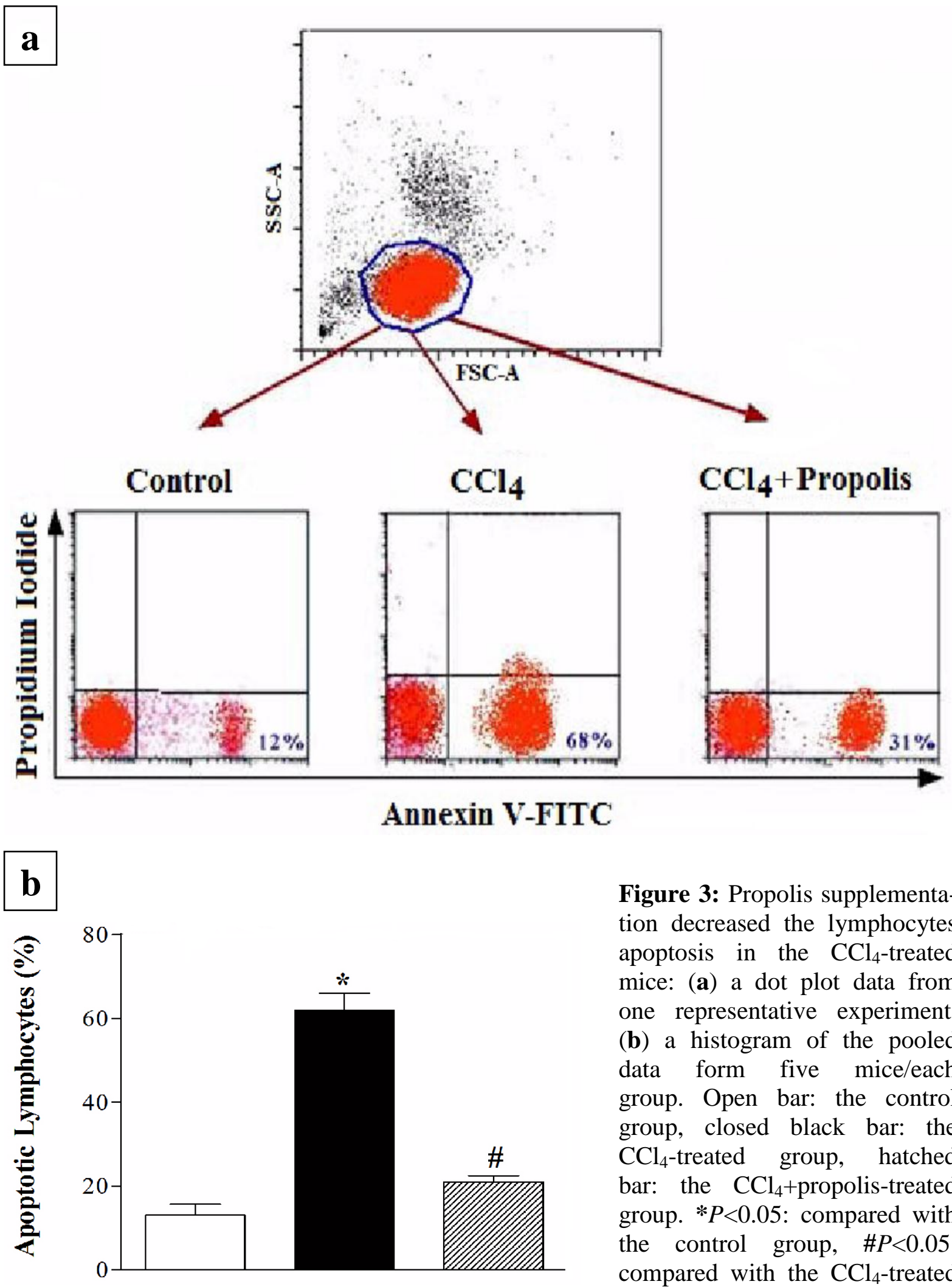

Figure 3: Propolis supplementation decreased the lymphocytes apoptosis in the $\mathrm{CCl}_{4}$-treated mice: (a) a dot plot data from one representative experiment, (b) a histogram of the pooled data form five mice/each group. Open bar: the control group, closed black bar: the $\mathrm{CCl}_{4}$-treated group, hatched bar: the $\mathrm{CCl}_{4}+$ propolis-treated group. ${ }^{*} P<0.05$ : compared with the control group, $\# P<0.05$ : compared with the $\mathrm{CCl}_{4}$-treated group.

the control mice (Figure 4c). However, supplementation of $\mathrm{CCl}_{4}$-treated mice with propolis restored significantly $(P<0.05)$ the chronic oxidative stress state that was mediated by $\mathrm{CCl}_{4}$ by increasing the level of total glutathione in the plasma. 

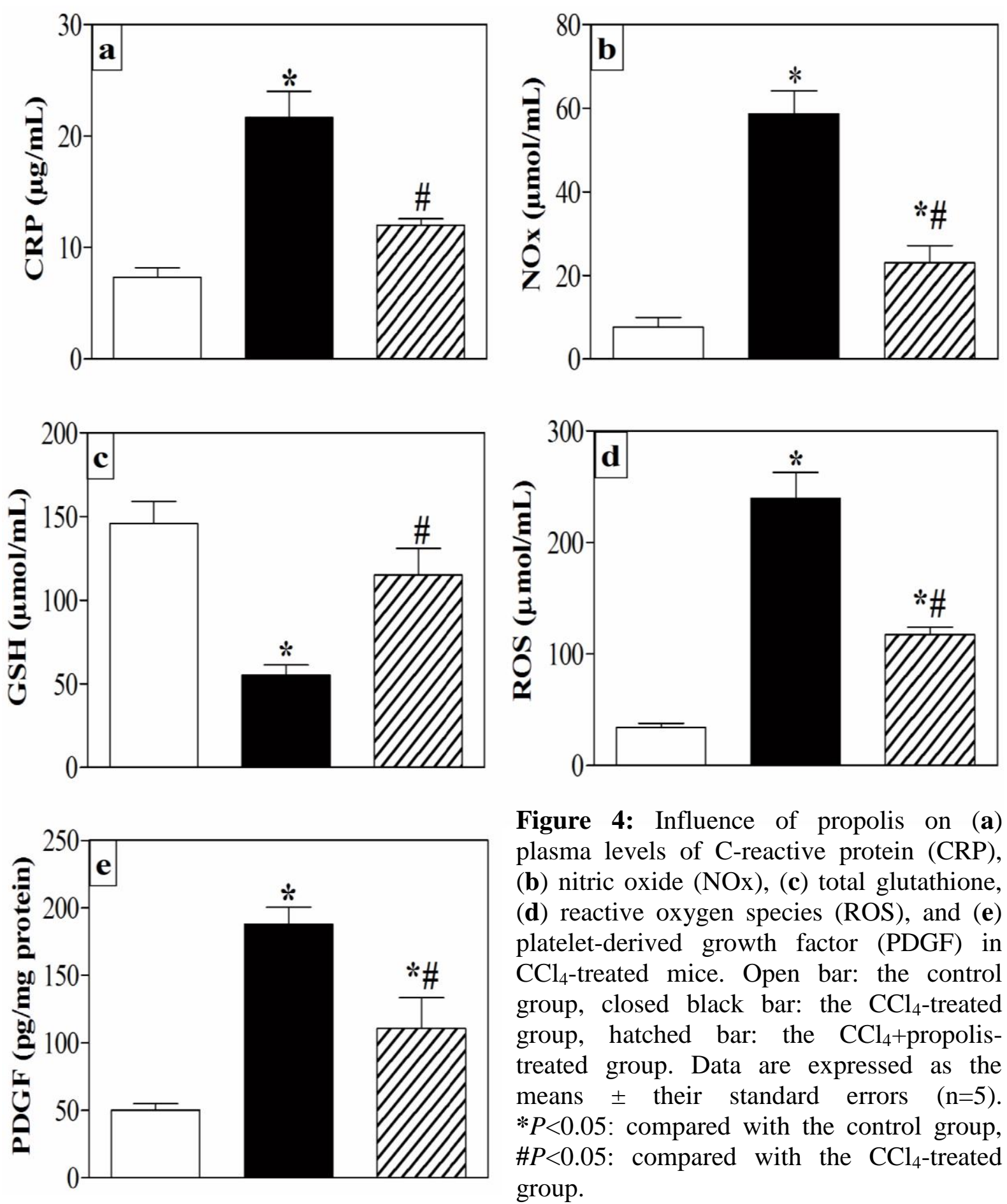

Figure 4: Influence of propolis on (a) plasma levels of C-reactive protein (CRP), (b) nitric oxide (NOx), (c) total glutathione, (d) reactive oxygen species (ROS), and (e) platelet-derived growth factor (PDGF) in $\mathrm{CCl}_{4}$-treated mice. Open bar: the control group, closed black bar: the $\mathrm{CCl}_{4}$-treated group, hatched bar: the $\mathrm{CCl}_{4}+$ propolistreated group. Data are expressed as the means \pm their standard errors $(n=5)$. $* P<0.05$ : compared with the control group, $\# P<0.05$ : compared with the $\mathrm{CCl}_{4}$-treated group.

Oral supplementation of propolis in mice with fibrotic liver restored the expression of collagen, survivin, STAT3, and STAT5

Figures "5a and c" showed the immunoblots for the collagen type I, survivin, and $\beta$-actin (loading control) in the liver lysates of control, $\mathrm{CCl}_{4}$-treated mice, and $\mathrm{CCl}_{4}+$ propolis-treated mice. The expression levels of the collagen type I and survivin

were normalized to the total $\beta$-actin protein levels (Figures $5 \mathrm{~b}$ and $\mathrm{d}$, respectively). The pooled data of five mice from each group revealed that the $\mathrm{CCl}_{4}$-treated mice exhibited a significant elevation in the expression level of the collagen type I (Figure 5b) and a significant decrease in the expression level of survivin (Figure 5d), as compared with that in the control 

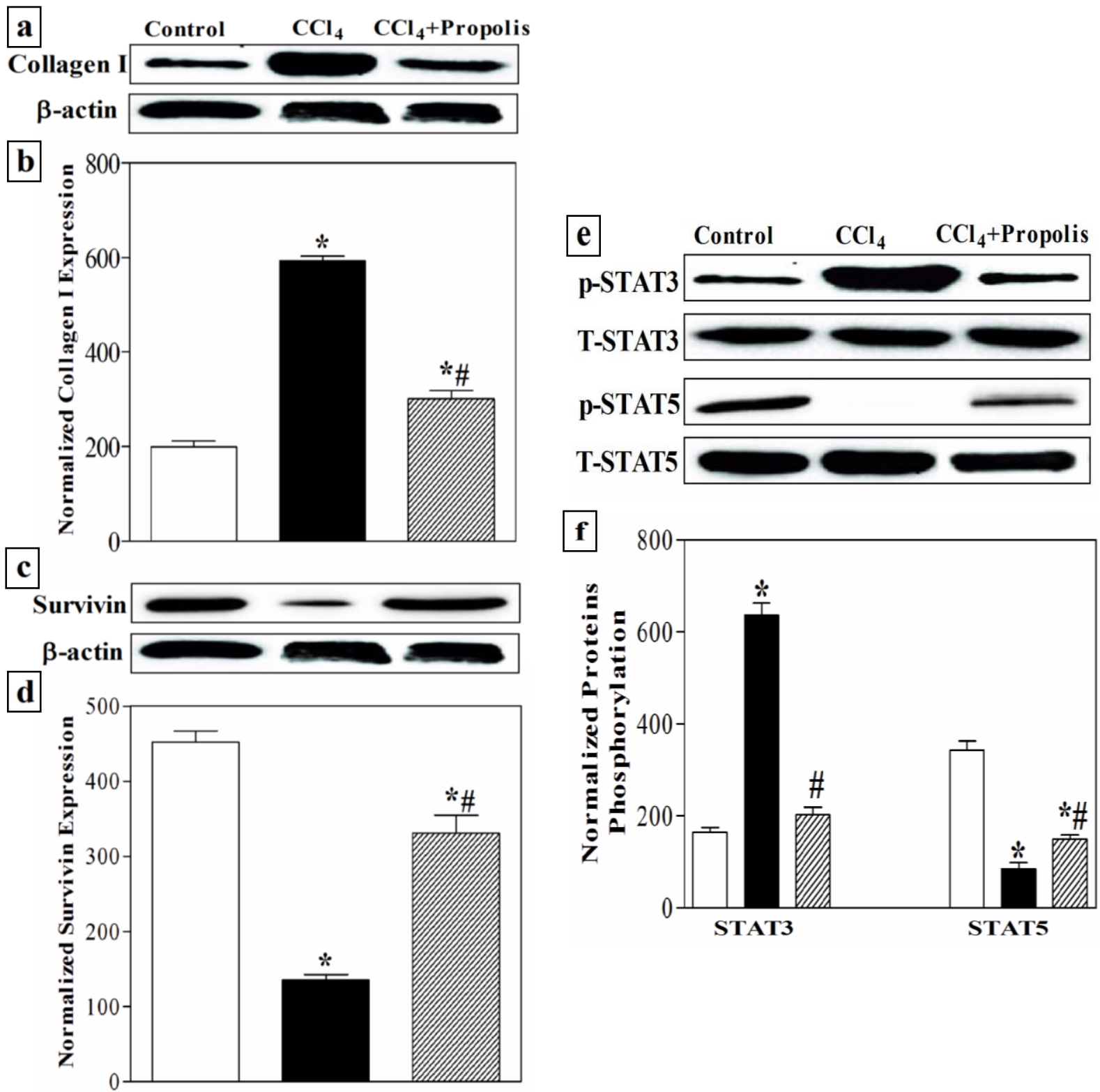

Figure 5: Propolis altered the expression of collagen I and survivin, and phosphorylation of signal transducer and activator of transcription 3 (STAT3) and STAT5, in $\mathrm{CCl}_{4}$-treated mice: (a, c, and e) the protein bands from one representative experiment for the expression of collagen I, survivin, $\beta$-actin (a house keeping gene), STAT3, and STAT5, (b, d, and f) accumulated data of the normalized value of each parameter of five mice from each group. The levels of phosphorylated STAT3 and phosphorylated STAT5 (p-STAT3 and p-STAT5, respectively) were normalized to the total STAT3 and total STAT5 (T-STAT3 and T-STAT5, respectively) protein levels. Open bar: the control group, closed black bar: the $\mathrm{CCl}_{4}$-treated group, hatched bar: the $\mathrm{CCl}_{4}+$ propolis-treated group. Data are expressed as the means \pm their standard errors $(\mathrm{n}=5)$. $* P<0.05$ : compared with the control group, $\# P<0.05$ : compared with the $\mathrm{CCl}_{4}$-treated group.

mice $(P<0.05)$. Interestingly, when $\mathrm{CCl}_{4}-$ treated mice were orally supplemented with propolis, they exhibited a significant downregulation of collagen type I expression and a significant upregulation of surviving expression compared with that in $\mathrm{CCl}_{4^{-}}$ treated mice $(P<0.05$, Figures $5 \mathrm{~b}$ and $\mathrm{d}$, respectively). The pooled data of five mice 
from each group revealed that the $\mathrm{CCl}_{4}$ treated group exhibited a significant increase in the phosphorylation of STAT3 and a significant decrease in the phosphorylation of STAT5 compared with that in the control group $(P<0.05$, Figures $5 \mathrm{e}$ and $\mathrm{f})$. After supplementation of the $\mathrm{CCl}_{4}$-treated mice with the propolis, the phosphorylation of STAT3 was significantly decreased and phosphorylation of STAT5 was significantly increased, as compared with that in the $\mathrm{CCl}_{4}$-treated group $(P<0.05$, Figure $5 \mathrm{f})$.

\section{DISCUSSION}

Natural antioxidants, such as propolis, play a vital role in improving the immune system through mechanisms that depend on oxidative stress; in turn, oxidative stress appears to play a major role in several human diseases ${ }^{[14]}$. The present study was designed to explore additional mechanistic protective effects of propolis in a mouse model of liver fibrosis. Our results revealed that the liver sections of the $\mathrm{CCl}_{4}$-treated group showed massive diffuse progressive histological alterations, loss of architecture with vacuolar degeneration and necrosis of hepatocytes, fatty changes in hepatocytes, sinusoidal dilatation, and dilated congested central vein together with fibrosis. The recorded pathological alterations can be attributed to the effect of $\mathrm{CCl}_{4}$ on the mitochondrial function of liver cells causing loss of $\mathrm{Ca}^{+2}$ from the mitochondria ${ }^{[15]}$. Propolis ethanolic extract successfully mitigated microscopic pathological changes of rabbit livers induced by $\mathrm{CCl}_{4}$ injection to be more or less similar to the control group $^{[16]}$. Kupffer cells are macrophages resident in the liver, necessary for hepatic homeostasis. When activated by danger signals, Kupffer cells recruit other immune cells such as monocytes and neutrophils to counteract diseased conditions initiating inflammation, fibrosis, angiogenesis, and repair ${ }^{[17]}$. Restorative macrophages derived from recruited monocytes facilitated the resolution of hepatic damage and fibrosis, when liver injury stops ${ }^{[1]}$. Our results revealed an increase in the number of
Kupffer cells observed in the $\mathrm{CCl}_{4}$-treated group, while in the $\mathrm{CCl}_{4}+$ propolis-treated group the number of the Kupffer cells returned to their normal levels. These data supported several previous studies that suggested that propolis has clinical potential as a natural anti-inflammatory agent ${ }^{[18]}$.

The results of the present study also showed that the percentage of apoptotic lymphocytes was increased in $\mathrm{CCl}_{4}$-treated group. Interestingly, treatment with propolis decreased the percentage of apoptotic lymphocytes. Chan et al. ${ }^{[19]}$ also reported that the Brazilian green propolis of southern Brazil (another source of propolis, where the major plant source is Baccharis dracunculifolia) exerted its inhibitory effect on the $\mathrm{CD}^{+}$T-helper cells through selectively induction of their apoptosis and not by the induction of the regulatory T-cells. The level of CRP (an inflammatory marker) was significantly increased in the current study in the $\mathrm{CCl}_{4}$-treated group and was significantly decreased in the $\mathrm{CCl}_{4}+$ propolis-treated group. Some antiinflammatory substances found in the propolis have been reported including caffeic acid, caffeic acid phenethyl ester, quercetin, naringenin, salicylic acid, apigenin, ferulic acid, and galangin ${ }^{[7]}$. The NOx is a crucial proinflammatory mediator (involved in the immune responses) and a highly active nitrogen species produced by inducible nitric oxide synthases ${ }^{[20]}$. Moreover, NOx reacts with superoxide anions to form a strong cytotoxic oxidant, namely peroxynitrite, which causes lipid peroxidation and cellular damage. The over production of NOx can cause hepatic injury, and the inhibition of NOx can reduce inflammatory damage ${ }^{[20]}$. Similarly, our results demonstrated that the level of $\mathrm{NOx}$ was increased in $\mathrm{CCl}_{4}$-treated group. Supplementation of $\mathrm{CCl}_{4}$-treated group with propolis restored the level of NOx to the normal values. The generation of ROS plays an important role in producing liver damage and initiating hepatic fibrogenesis through disrupting the cellular macromolecules and DNA, inducing necrosis and 
apoptosis of hepatocytes, and amplifying the inflammatory response ${ }^{[3]}$. ROS also stimulate the production of profibrogenic mediators from Kupffer cells and circulating inflammatory cells and directly activate hepatic stellate cells, resulting in the initiation of fibrosis ${ }^{[3]}$. Most interestingly, supplementation of $\mathrm{CCl}_{4}$-treated group with propolis alleviated the chronic oxidative stress state that was mediated by $\mathrm{CCl}_{4}$ through decreasing the level of plasma ROS. The ability of the propolis to reduce the oxidative stress and to inhibit the free radicals formation was probably due to its high content of the phenolics, and the ability of its phenolic compounds to donate hydrogen ions that can attack the free radicals and prevent the oxidation reactions in the cell ${ }^{[7]}$. Cellular glutathione is the chief antioxidant and redox regulator, and thus it is an important preventive agent for many diseases, including liver disease. It plays a key role in eliminating the reactive toxic metabolites of $\mathrm{CCl}_{4}{ }^{[21]}$. The results of the present study showed that the level of plasma total glutathione was significantly decreased in the $\mathrm{CCl}_{4}$-treated group, and was significantly increased in the $\mathrm{CCl}_{4}+$ propolistreated group. Bhadauria et al. ${ }^{[21]}$ also showed that $\mathrm{CCl}_{4}$ administration decreased the concentration of reduced glutathione in the liver tissues. Oral propolis administration restored the total glutathione level, which may in turn improve the detoxification of active metabolites of $\mathrm{CCl}_{4}$. Among cytokines, the most potent factor involved in the stimulation of HSC proliferation, differentiation, and migration is the platelet derived growth factors (PDGF) ${ }^{[22]}$. PDGF additionally promotes collagen production and deposition, and transforms HSCs into myofibroblasts. Inhibiting PDGF signalling decreases HSC proliferation, and alleviates liver fibrogenesis. Clinical studies also confirmed that the excessive activation of PDGF, and its downstream molecules, was associated with the degree of necroinflammation and fibrosis in the hepatic injury ${ }^{[22]}$. Thus, the PDGF signalling pathway plays an important role in the development of the liver fibrosis and its prognosis. Our results showed that the level of PDGF was significantly increased in the $\mathrm{CCl}_{4}$-treated group, and was significantly decreased in the $\mathrm{CCl}_{4}+$ propolistreated group. Activated HSCs express $\alpha$-smooth muscle actin and procollagen-I, and are considered to be the main source of collagen I and other fibrosis-deposited matrix proteins; thus, most antifibrotic therapies are designed to prevent HSCs activation and proliferation, or the release of their products ${ }^{[23]}$. Our results also demonstrated that $\mathrm{CCl}_{4}$-treated group that was supplemented with propolis showed reduction of chronic inflammation with a decrease in the deposition of type I collagen. Our results revealed that the $\mathrm{CCl}_{4}-$ treated mice exhibited a decrease in the expression of survivin (a member of apoptosis inhibitor proteins family) and the phosphorylation of STAT5, but exhibited an obvious increase in the phosphorylation of STAT3, while oral supplementation of the $\mathrm{CCl}_{4}$-treated mice with propolis modulated significantly these changes. Aydin et al. ${ }^{[2]}$ found that the activation of STAT3 transcription through IL-6 production participated in inflammation, tumourigenesis, and autophagy in hepatitis $\mathrm{C}$ virus infection. In addition, Friedbichler et $a{ }^{[25]}$ demonstrated that the loss of STAT5 activity is associated with higher susceptibility to liver fibrosis and cancer, suggesting that hepatic STAT5 has a protective role in mouse models of chronic liver disease. Interestingly, supplementation of $\mathrm{CCl}_{4}$-treated animals with propolis decreased the phosphorylation of STAT3 and increased the phosphorylation of STAT5. Búfalo et $a l^{[26]}$ also reported the protective effects of propolis against bacterial endotoxins-mediated inflammation through its inhibitory effects on the activation of mitogen-activated protein kinases in macrophages. In conclusion, propolis alleviated liver fibrosis in mice treated with $\mathrm{CCl}_{4}$ though controlling the STAT-3/STAT-5 phosphorylation and survivin expression in liver tissues, as well 
as decreasing the systemic inflammation and oxidative stress.

\section{ACKNOWLEDGEMENTS}

The authors extend their appreciation to the Laboratory of Immunology and Molecular Physiology, Faculty of Science, Assiut University, for funding this work.

\section{CONFLICT OF INTEREST}

The authors declare no conflict of interest.

\section{AUTHORS' CONTRIBUTIONS}

EAS carried out all the experiments and helped in the figure preparation. HW participated in the study design. KAH participated in the study design and revised the manuscript. GB put the study design, participated in all the experiments, performed the statistical analysis, and drafted the manuscript. All authors read and approved the final manuscript.

\section{REFERENCES}

[1] Wree, A.; Eguchi, A.; McGeough, M. D. et al. (2014). NLRP3 inflammasome activation results in hepatocyte pyroptosis, liver inflammation, and fibrosis in mice. Hepatology, 59(3): 898-910.

[2] Friedman, S. L. (2008). Hepatic fibrosis - overview. Toxicology, 254(3): 120-129.

[3] Gäbele, E.; Brenner, D. A. and Rippe, R. A. (2003). Liver fibrosis: signals leading to the amplification of the fibrogenic hepatic stellate cell. Front Biosci, 8: d69-77.

[4] Bruck, R.; Hershkoviz, R.; Lider, O. et al. (1996). Inhibition of experimentally-induced liver cirrhosis in rats by a nonpeptidic mimetic of the extracellular matrix-associated Arg-Gly-Asp epitope. Journal of hepatology, 24(6): 731-738.

[5] Hashem, A. S.; Taha, N. M.; Mandour, A. A. et al. (2016). Hepatoprotective effect of silymarin and propolis in chemically induced chronic liver injury in rats. Alexandria
Journal for Veterinary Sciences, 49: 35-43.

[6] Aldaba-Muruato, L. R.; Moreno, M. G.; Shibayama, M. et al. (2012). Protective effects of allopurinol against acute liver damage and cirrhosis induced by carbon tetrachloride: modulation of $\mathrm{NF}-\kappa \mathrm{B}$, cytokine production and oxidative stress. Biochim Biophys Acta, 1820(2): 65-75.

[7] Sforcin, J. M. and Bankova, V. (2011). Propolis: is there a potential for the development of new drugs? J Ethnopharmacol, 133: 253-260.

[8] Jerz, G.; Elnakady, Y. A.; Braun, A. et al. (2014). Preparative massspectrometry profiling of bioactive metabolites in Saudi-Arabian propolis fractionated by high-speed countercurrent chromatography and off-line atmospheric pressure chemical ionization mass-spectrometry injection. $\mathbf{J}$ Chromatogr A., 1347: 17-29.

[9] Hidaka, M.; Eguchi S.; Takatsuki, M. et al. (2016). The Kupffer cell number affects the outcome of living donor liver transplantation from elderly donors. Transplant Direct, 2(8): e94 (DOI: 10.1097/TXD. 0000000000000608).

[10] Eruslanov, E. and Kusmartsev, S. (2010). Identification of ROS using oxidized DCFDA and flow-cytometry. Methods Mol Biol, 594: 57-72.

[11] Ono, H.; Sakamoto, A. and Sakura, N. (2000). Plasma total glutathione concentrations in epileptic patients taking anticonvulsants. Clin Chim Acta, 298: 135-143.

[12] Sin, K.-K.; Chan, C. P.-Y.; Leung, W.-M. et al. (2006). Fluorogenic nanocrystals for highly sensitive detection of C-reactive protein. IEE Proc-Nanobiotechnol, 153(3): 54-58.

[13] Badr, G.; Al-Sadoon, M. K.; El-Toni, A. M. et al. (2012). Walterinnesia egyptian venom combined with silica nanoparticles enhances the functioning of normal lymphocytes 
through $\mathrm{PI} 3 \mathrm{~K} / \mathrm{AKT}, \mathrm{NF} \kappa \mathrm{B}$ and ERK signaling. Lipids Health Dis, 11: 27 (doi:10.1186/1476-511X-11-27).

[14] Al-Ghamdi, A. A.; Badr, G.; Hozzein, W. N. et al. (2015). Oral supplementation of diabetic mice with propolis restores the proliferation capacity and chemotaxis of $\mathrm{B}$ and $\mathrm{T}$ lymphocytes towards CCL21 and CXCL12 by modulating the lipid profile, the pro-inflammatory cytokine levels and oxidative stress. BMC Immunol, 16: $54 \quad(10.1186 / \mathrm{s} 12865-$ 015-0117-9).

[15] Albano, E.; Bellomo, G.; Carini, R. et al. (1985). Mechanisms responsible for carbon tetrachloride-induced perturbation of mitochondrial calcium homeostasis. FEBS Lett, 192(2): 184188.

[16] Jabir, F. A. and Hadi, N. K. (2014). Study of the hepatoprotective activity of ethanol extract of local propolis in rabbits. Al-Qadisiah Medical Journal, 10(2): 37-45.

[17] Sato, K.; Kennedy, L.; Liangpunsakul, S. et al. (2019). Intercellular communication between hepatic cells in liver diseases. Int J Mol Sci, 20(9): 2180 (DOI: 10.3390/ijms20092180).

[18] Alqarni, A. M.; Niwasabutra, K.; Sahlan, M. et al. (2019). Propolis exerts an anti-inflammatory effect on PMA-differentiated THP-1 cells via inhibition of purine nucleoside phosphorylase. Metabolites, 9(4): E75 (DOI: 10.3390/metabo9040075).

[19] Chan, G. C. F.; Cheung, K. W. and Sze, D. M. Y. (2013). The immunomodulatory and anticancer properties of propolis. Clin Rev Allergy Immunol, 44(3): 262-273.

[20] Jaeschke, H.; Gores, G. J.; Cederbaum,
A. I. et al. (2002). Mechanisms of hepatotoxicity. Toxicol Sci, 65(2): 166-176.

[21] Bhadauria, M.; Nirala, S. K and Shukla, S. (2007). Duration-dependent hepatoprotective effects of propolis extract against carbon tetrachlorideinduced acute liver damage in rats. Advances in therapy, 24: 11361145.

[22] Ying, H.-Z.; Chen, Q.; Zhang, W.-Y. et al. (2017). PDGF signalling pathway in hepatic fibrosis pathogenesis and therapeutics. Mol Med Rep, 16(6): 7879-7889.

[23] Montes, G. S. and Junqueira, L. C. (1991). The use of the Picrosirius polarization method for the study of the biopathology of collagen. Mem Inst Oswaldo Cruz, 86(3): 1-11.

[24] Aydin, Y.; Kurt, R.; Song, K. et al. (2019). Hepatic stress response in $\mathrm{HCV}$ infection promotes STAT3mediated inhibition of HNF4A-miR122 feedback loop in liver fibrosis and cancer progression. Cancers (Basel), 11(10): E1407 (DOI: 10.3390/ cancers11101407).

[25] Friedbichler, K.; Themanns, M.; Mueller, K. M. et al. (2012). Growthhormone-induced signal transducer and activator of transcription 5 signaling causes gigantism, inflammation, and premature death but protects mice from aggressive liver cancer. Hepatology, 55(3): 941-952.

[26] Búfalo, M. C.; Ferreira, I.; Costa, G. et al. (2013). Propolis and its constituent caffeic acid suppress LPS-stimulated pro-inflammatory response by blocking $\mathrm{NF}-\kappa \mathrm{B}$ and MAPK activation in macrophages. J Ethnopharmacol, 149: 84-92.

\section{How to cite this article:}

Sayed, E. A.; Waly, H.; Hassan, K. A. and Badr, G. (2020). Propolis improved the hepatic architecture by controlling STAT-3 and STAT-5 phosphorylation, and survivin expression in a mouse model of liver fibrosis. Egyptian Journal of Zoology, 73: 39-52 (DOI: 10.12816/ejz.2020.26777. 1027). 


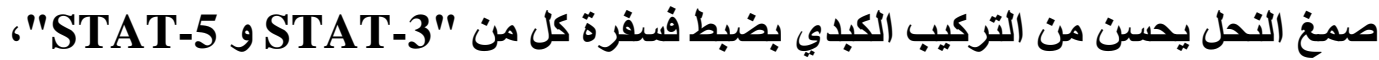 وتعبير "Survivin" في نموذج الفئران المصابة بالتليف الكبدي
}

\author{
ايمان عبده سيد، حنان والي، خديجة عبدالحمبد حسن، جمال بلر \\ قسم علم الحيوان، كلية العلوم، جامعة أسيوط، أسيوط، جمهورية مصر العبل، العربية
}

لصمغ النحل الكثير من الخصائص الحيوية والفارماكولوجية. أجريت الدراسة الحالية لتقييم الدور الوقائي لصمغ النحل

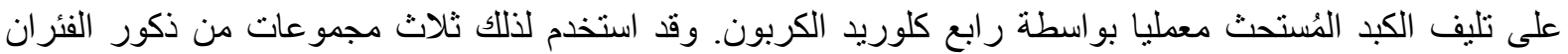

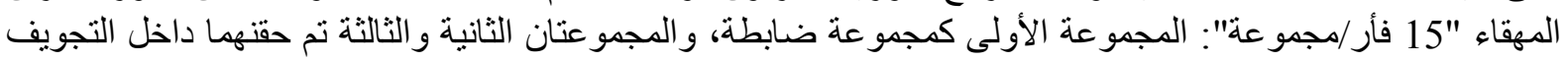

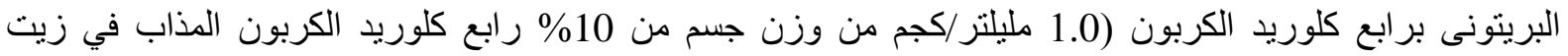

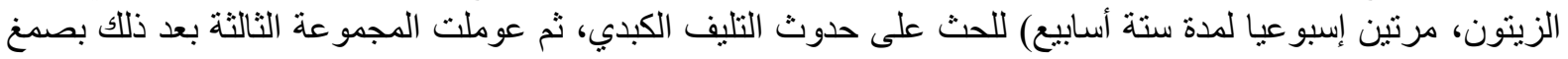

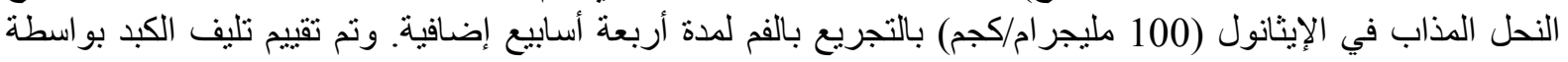

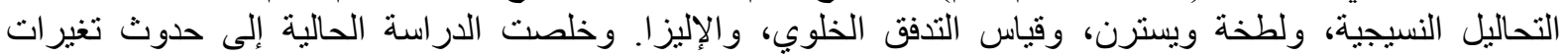

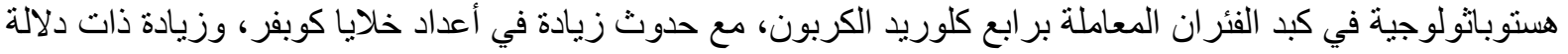

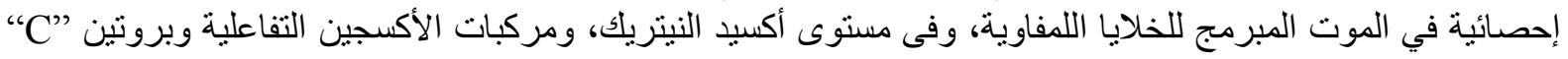

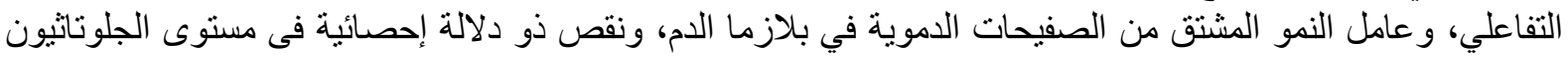

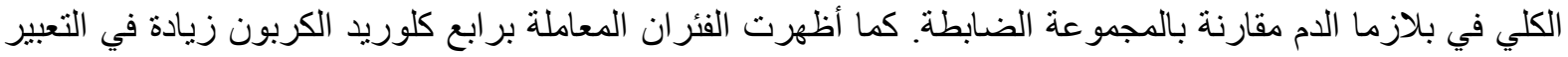

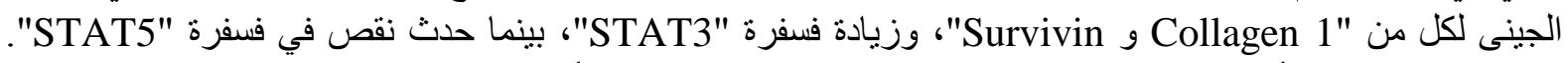

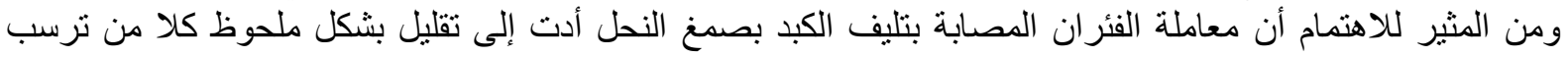

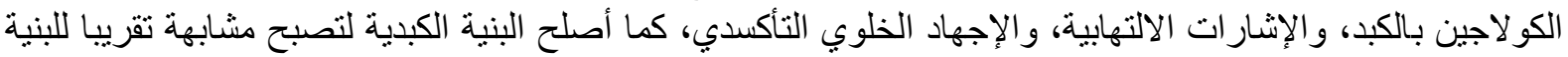

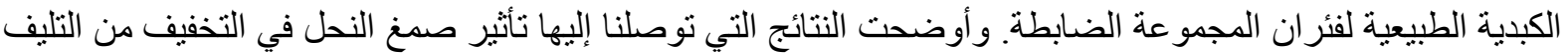

